\title{
10. \\ Rapporti familiari a Mutina e nel suo agro tra III e V secolo d.C.: considerazioni alla luce della documentazione epigrafica
}

\author{
Manuela Mongardi
}

DOI - 10.7359/764-2016-mong

ABSTRACT - The paper focuses on the collection and analysis of the epigraphic evidence in Mutina dating from the $3^{\text {rd }}$ century $\mathrm{AD}$ to the $5^{\text {th }}$ century $\mathrm{AD}$, with particular attention to funerary inscriptions. For the period between the half of the $3^{\text {rd }}$ century $\mathrm{AD}$ and the half of the $4^{\text {th }}$ century AD it can be noted a significant presence of military officers and exponents of the bureaucracy, who often reused ancienter proconnesian marble sarcophagi for themselves or their relatives. Almost all the inscriptions contain an explicit indication of the family relationships among the persons mentioned so it is possible to reconstruct family units which sometimes include ancestors until the second generation. An interesting case is that of the vir consularis L. Nonius Verus, for whom epigraphic documents allow to reconstruct more than a family unit. In fact there are three inscriptions which have been traditionally related to L. Nonius Verus and mention three different wifes $(C I L$ $\mathrm{XI} 831,832,1017)$; in particular two of them surely concern the vir consularis whereas the third one could be attributed to a namesake relative.

KEYWORDS - Family relationships, funerary epigraphy, L. Nonius Verus, Mutina, $3^{\text {rd }}-5^{\text {th }}$ century AD. Epigrafia funeraria, legami familiari, L. Nonius Verus, Mutina, III-V secolo d.C.

La colonia romana di Mutina sorse in una posizione itineraria di rilievo, al centro dell'asse della via Aemilia ed in collegamento con Roma mediante tres viae ${ }^{1}$ nonché coll'area veneta ed alto-adriatica, attraverso cui si raggiungevano agevolmente il Noricum e la Pannonia ${ }^{2}$. In particolare, il perdurare tra III e IV secolo d.C. dell'importanza della via Aemilia come nodo stradale per le comunicazioni e per il passaggio di truppe dirette alle province nord-occidentali è evidenziato dall'interesse imperiale per la sua

1 Cic. Phil. XII 22.

2 Sulla viabilità di Mutina si veda ad esempio Campagnoli 2006. 
manutenzione, testimoniato dal rinvenimento di numerosi miliari, sette dei quali provenienti proprio dal territorio modenese ${ }^{3}$, nonché di una lastra celebrativa del restauro nel 259 d.C. del ponte sul fiume Secchia, posto nel tratto della via Aemilia che collegava Mutina e Regium Lepidi ${ }^{4}$.

Quanto alla città, a partire dalla metà del III secolo d.C., in concomitanza con la grave crisi economico-politica che investì l'impero, essa visse un periodo di decadenza e di progressivo decremento demografico ${ }^{5}$. Le fonti letterarie la menzionano, dopo un lungo silenzio risalente agli scontri tra Otone e Vitellio nel 69 d.C. ${ }^{6}$, in occasione del conflitto nel 312 d.C. tra Costantino e Massenzio, allorquando quest'ultimo inviò distaccamenti di truppe in alcuni centri dell'Italia settentrionale, tra cui Mutina, che, tuttavia, dopo un breve assedio, si consegnò a Costantino ${ }^{7}$, ottenendone probabilmente i favori e vivendo così un breve periodo di ripresa economica, documentato anche dalla presenza di militari e di alti funzionari legati alla corte imperiale, nonché dall'aumento del circolante divisionale ${ }^{8}$. Dal tardo IV secolo d.C. la città subì una contrazione, con fenomeni di «ruralizzazione» di alcune aree urbane e col progressivo abbandono del settore orientale e la successiva crescita del suburbio occidentale, che diverrà il nuovo polo religioso e civile della civitas altomedievale 9 ; tale evoluzione trova riflesso in una celebre epistola di Ambrogio, in cui, seppur con toni apocalittici che riprendono un topos letterario tipico delle consolationes, le città emiliane e tra queste Mutina - vengono definite semirutarum urbium cadavera ${ }^{10}$.

\section{LA DOCUMENTAZIONE EPIGRAFICA}

La documentazione epigrafica relativa a Mutina e al suo ager consta attualmente di 265 attestazioni, di cui 145 di natura sepolcrale. In particolare, al periodo compreso tra III e V secolo d.C. sono da riferire 30 iscrizioni ${ }^{11}$,

3 CIL XI 6651 (Massenzio), 6648 e 6654 (Costantino), 6652 (Costantino, Licinio e i Cesari Crispo, Licinio II e Costantino II), 6647 (Magnenzio); AÉ 1983, 418 (Giuliano), $A E ́$ 1985, $411=1995,518=2003,663$ (Valentiniano e Valente).

4 CIL XI $826=6648$ a.

5 Gelichi - Malnati - Ortalli 1986, 557-558.

6 Tac. hist. II 52-54.

7 Paneg. 4 [10], 27, 1-3.

8 Giordani 2000, 428 .

9 Cattani 1998, 41-43.

10 Ambr. epist. II 8, 3.

11 Sono state escluse dalla rassegna: cinque iscrizioni genericamente riferite al IIIII secolo d.C. (CIL XI 819, 882, 886, 913, 6930d); tre epigrafi datate tra la fine del II e gli 
pari all' $11,3 \%$ del totale, per la maggior parte funerarie. Esulano da questa tipologia, oltre ai già citati miliari e all'iscrizione relativa al rifacimento del ponte sul Secchia: due basi iscritte con dedica, rispettivamente, a $\mathrm{Nu}$ meriano e a Flavio Valerio Costanzo ${ }^{12}$ rinvenute in rua Pioppa, in un'area da identificare plausibilmente col Caesareum ${ }^{13}$; una dedica frammentaria su colonna forse da riferire ai Cesari figli di Costantino nota unicamente da tradizione manoscritta ${ }^{14}$; una lastra ${ }^{15}$, genericamente datata al IV-V secolo d.C., con la raffigurazione di due pesci e cinque pani sormontata dalla parola greca in caratteri latini syntropbion - banchetto in comune -, che in origine potrebbe aver avuto un valore topografico-funzionale di localizzazione di un elemento liturgico ed essere stata solo in seguito riutilizzata in una tomba ${ }^{16}$ piuttosto che riferirsi, come supposto da F. Rebecchi ${ }^{17}$, all'iscrizione sepolcrale di un Syntrophion.

Il nucleo di testimonianze più consistente - costituito da 15 attestazioni, cui si aggiungono due iscrizioni assai lacunose su sarcofago ${ }^{18}$ e un frammento di stele datato al III-IV secolo d.C. rinvenuto a Nonantola, località Redù su cui si conserva parzialmente un'epigrafe in prosa strutturata a imitazione di quelle elegiache ove è il defunto a parlare ${ }^{19}$ - è, come accennato, quello delle iscrizioni sepolcrali (Tab. 1).

inizi del III secolo d.C. (CIL XI 819; CIL XI 911 = CLE 1181; AÉ 1945, 60; CIL XI $6926=$ CLE 2013); un'iscrizione in onore di Salvius Satrianus Minucius (PIR ${ }^{2}$ S 147) rinvenuta a S. Agata Bolognese (CIL XI 833) e probabilmente non pertinente all'ager Mutinense (Jacques 1983, 142-143, nr. 60); un cippo da Montebaranzone con dedica ad Ercole e Giove o ad Hercules Iovius da parte di Betia Laudice (AÉ 1976, 224), riferito da F. Rebecchi a fine II - inizi III secolo d.C. (Rebecchi 1969, 273) ma probabilmente databile entro il I secolo d.C. (Corti 2012, 22) o, meglio, tra la fine del I e gli inizi del II secolo d.C. (ringrazio la professoressa A. Donati per il consulto cronologico).

12 CIL XI 827 e 828.

13 Giordani 2000, 428-429.

14 CIL XI 6655.

15 CIL XI $943=$ ILCV 1572.

16 Sastre de Diego 2011, 68-69.

17 Rebecchi 1986, 922.

18 Si tratta di un frammento della fronte di un sarcofago di marmo del III secolo d.C. su cui si leggono solo le due lettere iniziali Q F[---] vel E[---] rinvenuto nel Fondo San Pietro a S. Felice sul Panaro (Calzolari 1984, 27) e di un sarcofago a cassapanca datato all'ultimo quarto del III secolo d.C. rinvenuto in piazza Matteotti, in corrispondenza della necropoli W di Mutina, della cui iscrizione, erasa presumibilmente in occasione di un reimpiego tardoantico, si conserva solo l'adprecatio agli dei Mani (Giordani 2005)

19 CIL XI 932; Giordani - Ricci 2005. 


\begin{tabular}{|c|c|c|c|c|c|}
\hline 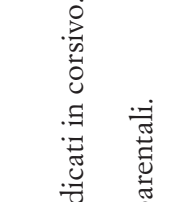 & 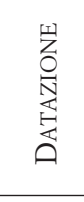 & $\begin{array}{l}\dot{j} \\
\dot{\vec{G}} \\
\dot{G}\end{array}$ & $\begin{array}{l}\dot{U} \\
\dot{\vec{g}} \\
\dot{\theta}\end{array}$ & 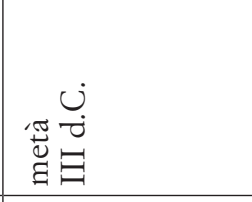 & 鸹岂 \\
\hline 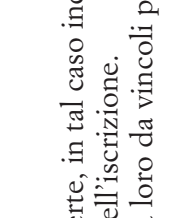 & $\begin{array}{l}\text { 离 } \\
\text { 商 } \\
\text { 怘 }\end{array}$ & 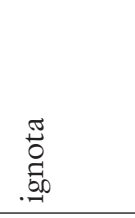 & 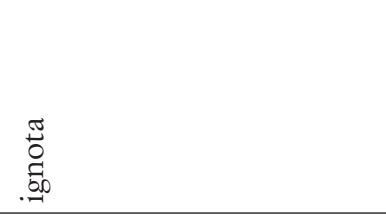 & 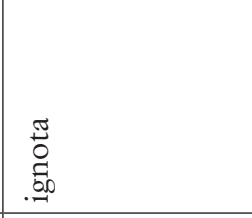 & 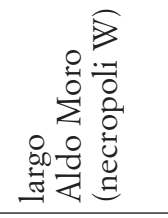 \\
\hline 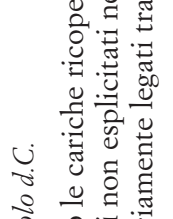 & 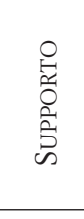 & $\begin{array}{l}8 \\
0 \\
0 \\
.50 \\
.00\end{array}$ & $\begin{array}{l}8 \\
\stackrel{8}{0} \\
.000 \\
.00\end{array}$ & $\frac{\ddot{U}}{\tilde{\omega}}$ & 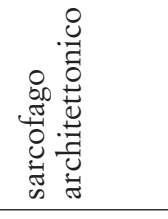 \\
\hline 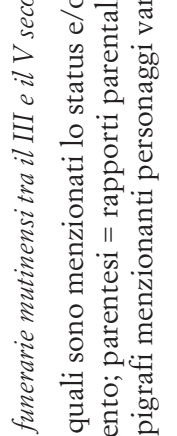 & 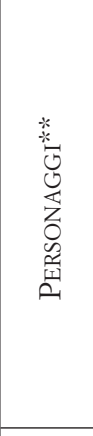 & 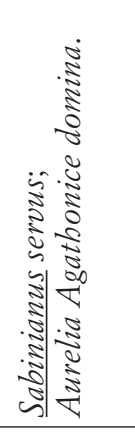 & 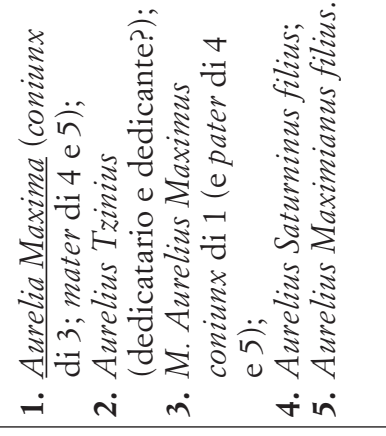 & 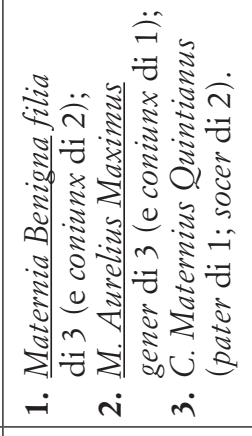 & 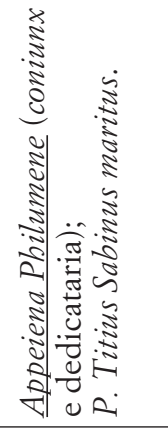 \\
\hline 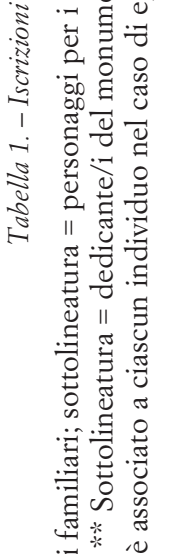 & 急 & 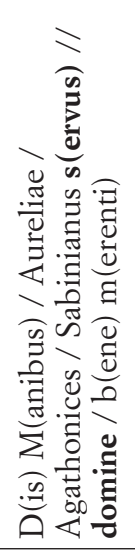 & 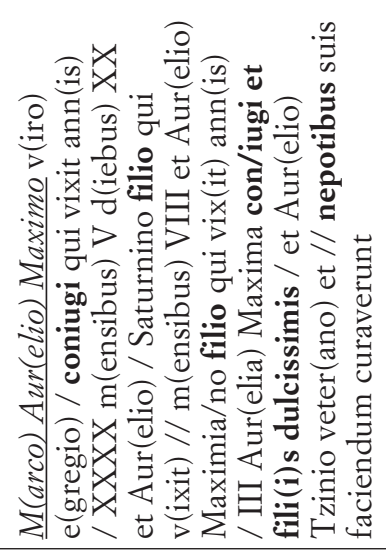 & 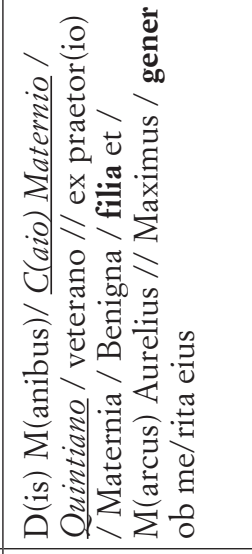 & 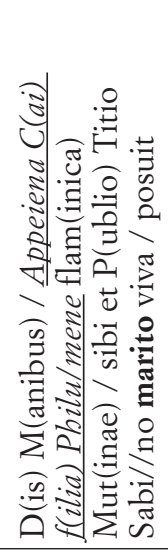 \\
\hline 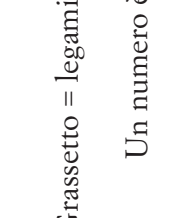 & 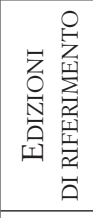 & 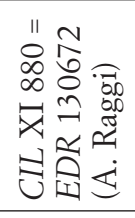 & 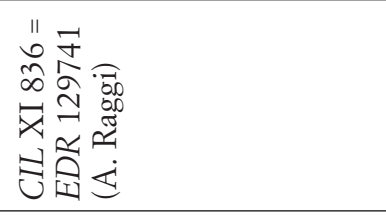 & 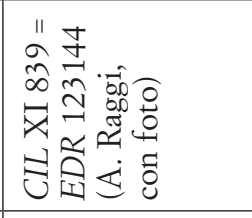 & 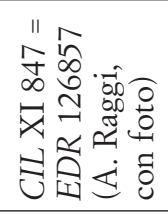 \\
\hline & $z$ & - & $N$ & n & $\sigma$ \\
\hline
\end{tabular}




\begin{tabular}{|c|c|c|c|c|}
\hline $\begin{array}{l}\dot{U} \\
\stackrel{\sigma}{0} \\
\Xi \Xi \\
\Xi\end{array}$ & 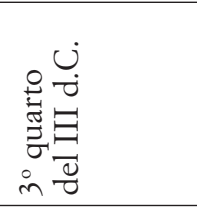 & 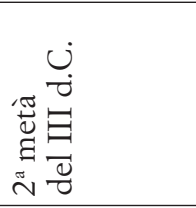 & 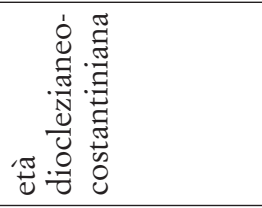 & 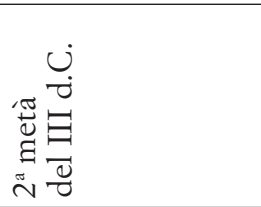 \\
\hline 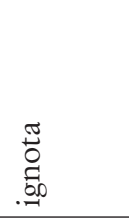 & 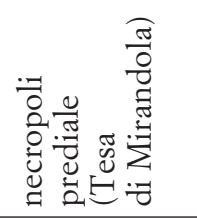 & 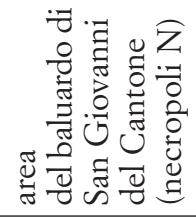 & 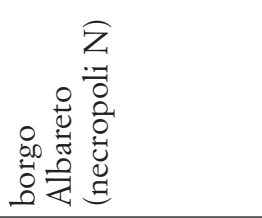 & 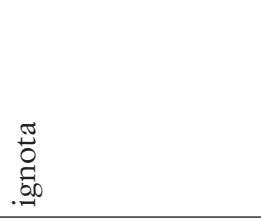 \\
\hline 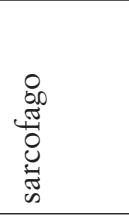 & 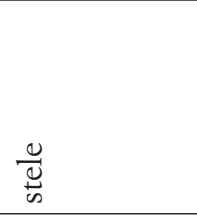 & 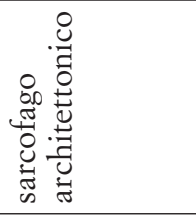 & 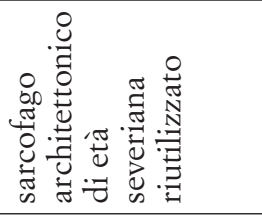 & 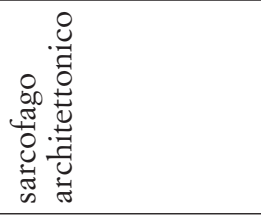 \\
\hline 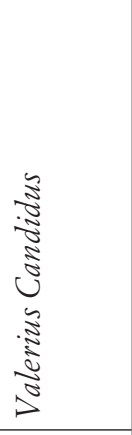 & 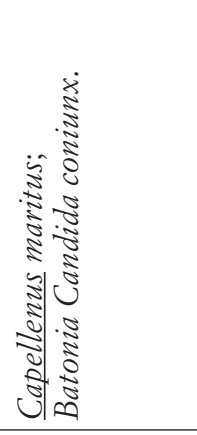 & 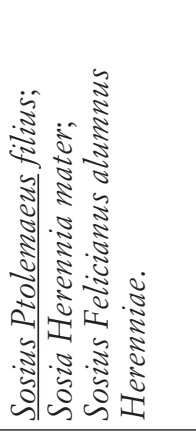 & 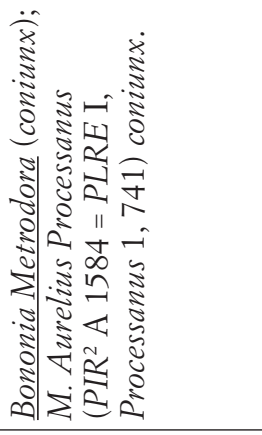 & 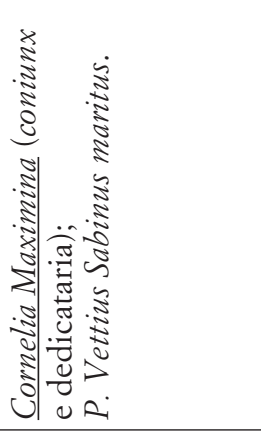 \\
\hline 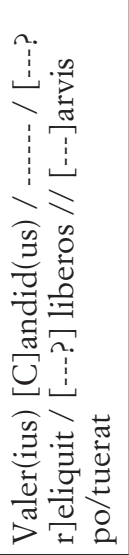 & 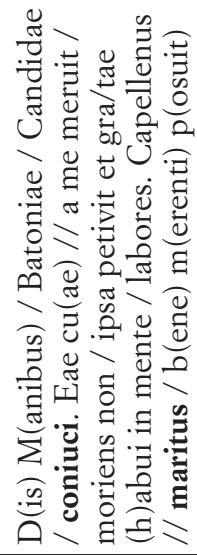 & 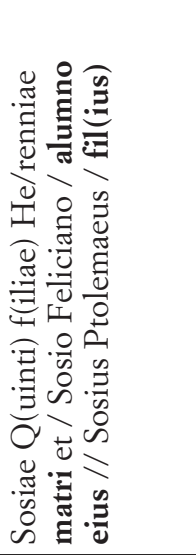 & 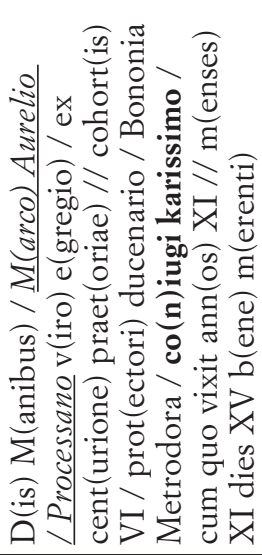 & 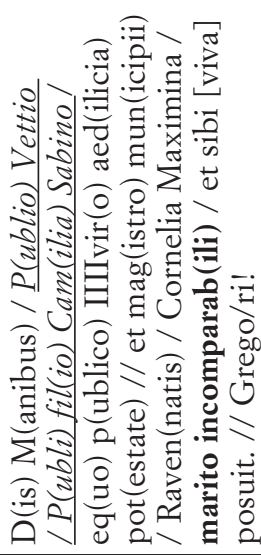 \\
\hline 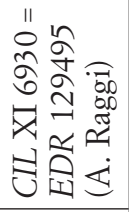 & 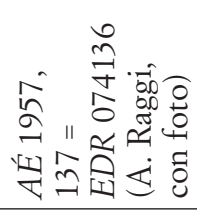 & 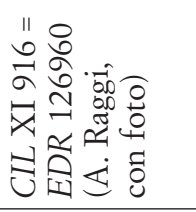 & 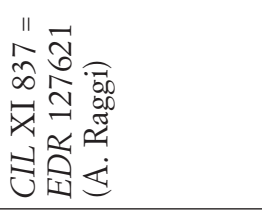 & 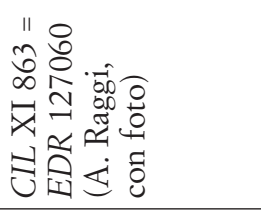 \\
\hline in & 0 & $r$ & $\infty$ & $a$ \\
\hline
\end{tabular}




\begin{tabular}{|c|c|c|c|}
\hline 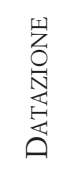 & 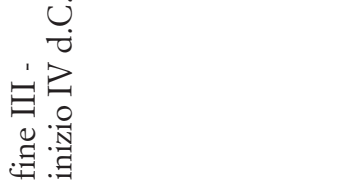 & 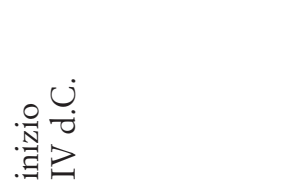 & 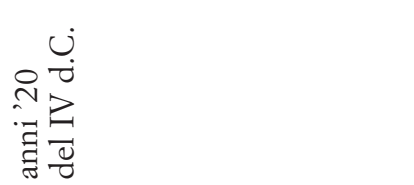 \\
\hline 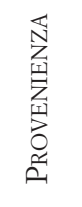 & $\begin{array}{l}\underset{\pi}{0} \\
\stackrel{0}{0} \\
.0-6 \\
.\end{array}$ & 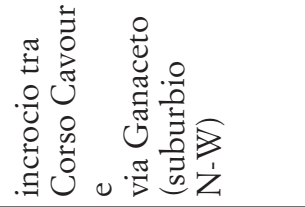 & 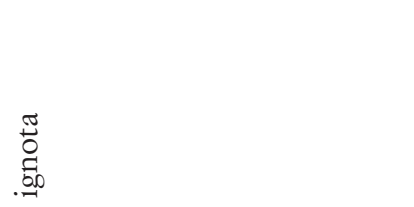 \\
\hline 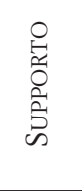 & $\frac{\vec{d}}{\stackrel{\vec{w}}{\infty}}$ & 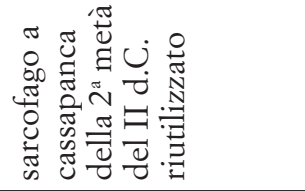 & 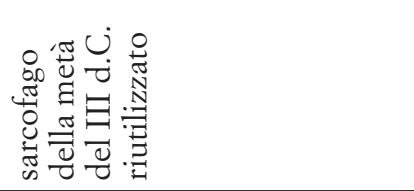 \\
\hline 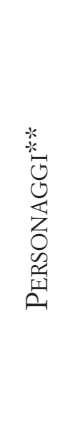 & 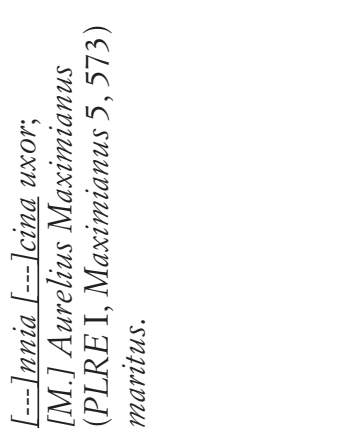 & 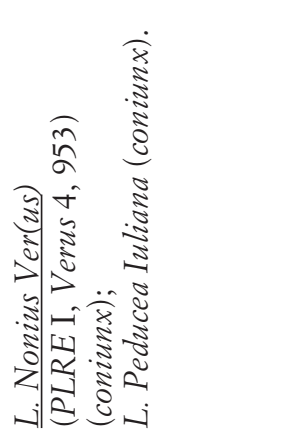 & 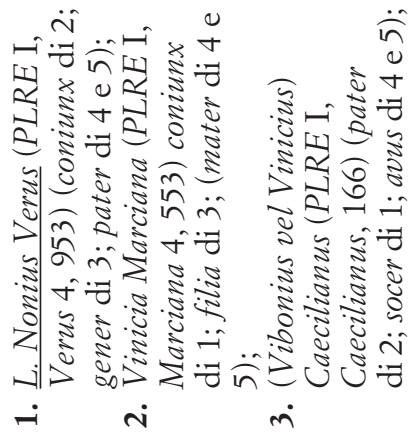 \\
\hline 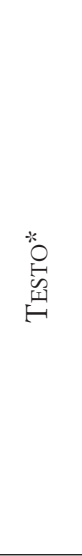 & 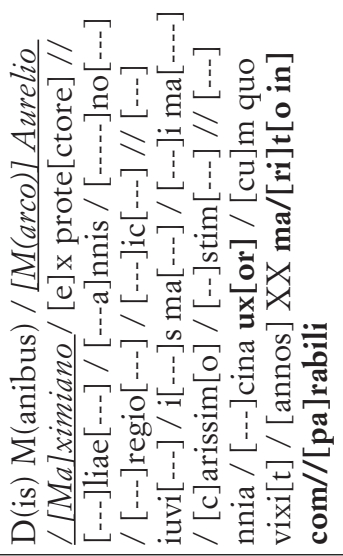 & 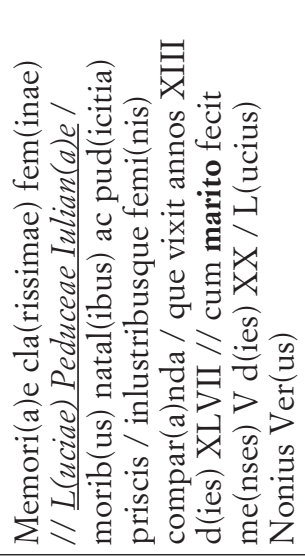 & 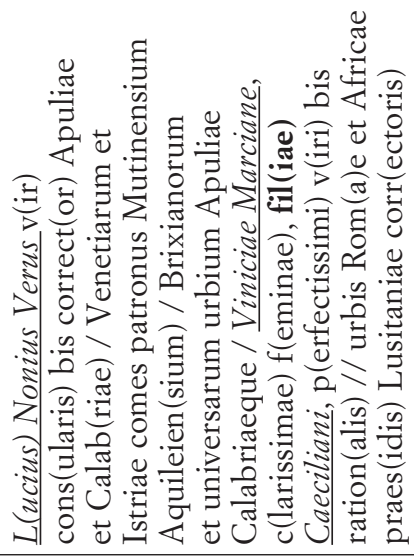 \\
\hline 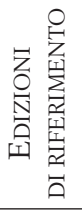 & 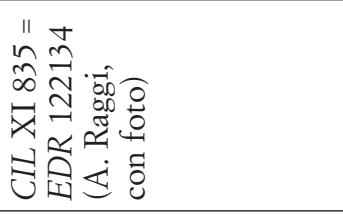 & 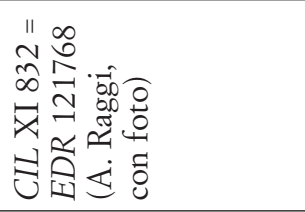 & 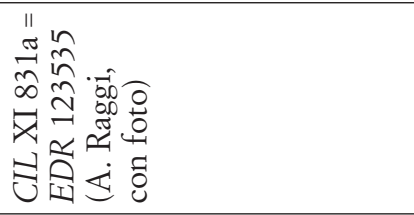 \\
\hline Z & 으 & $=$ & $\simeq$ \\
\hline
\end{tabular}




\begin{tabular}{|c|c|c|}
\hline & & 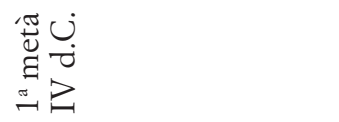 \\
\hline & & 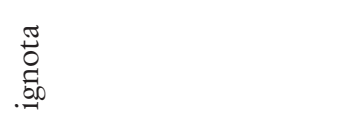 \\
\hline & & 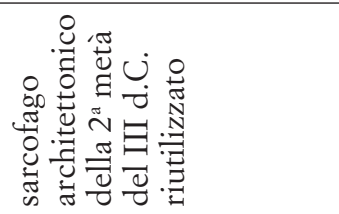 \\
\hline 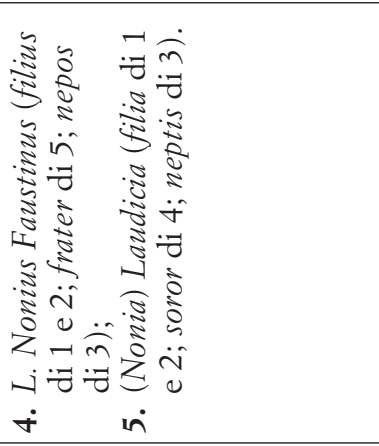 & & 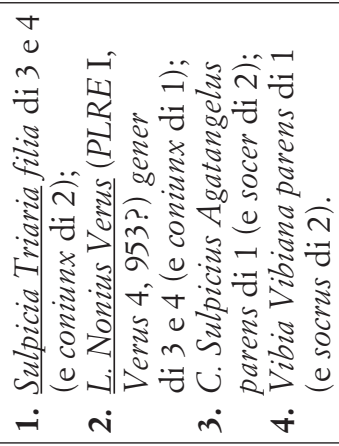 \\
\hline 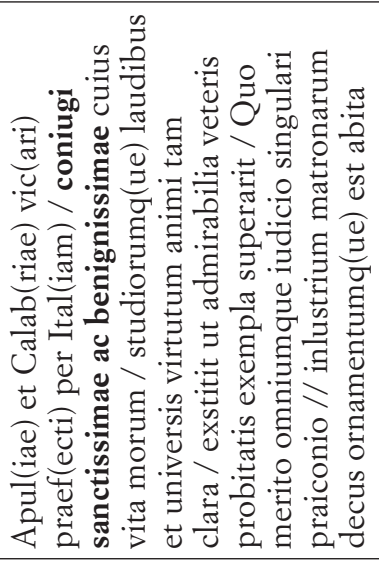 & 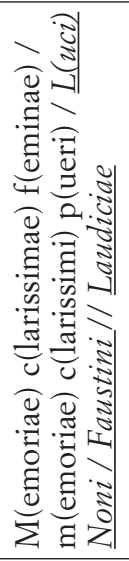 & 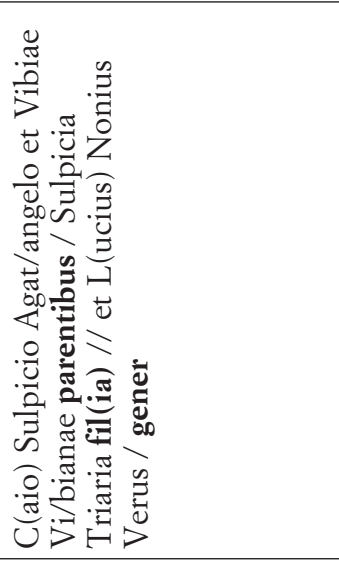 \\
\hline & 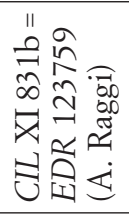 & 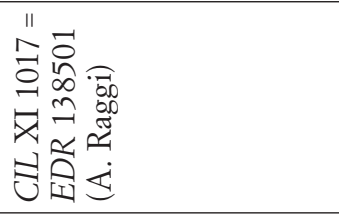 \\
\hline & & $\approx$ \\
\hline
\end{tabular}

La famiglia tardoantica. Società, diritto, religione - A cura di V. Neri e B. Girotti - Milano, LED, 2016 http://www.ledonline.it/index.php/Erga-Logoi/pages/view/qel-5-famiglia-tardoantica 


\begin{tabular}{|c|c|c|}
\hline 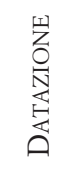 & 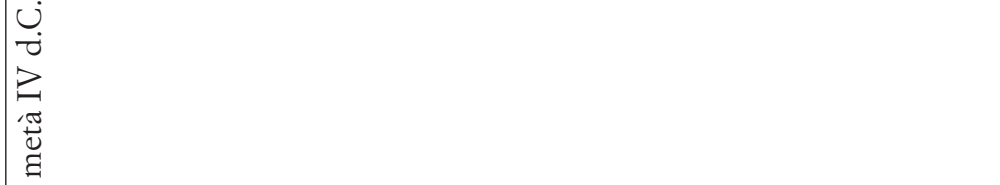 & $\begin{array}{l}\dot{U} \\
\ddot{D} \\
\ddot{1} \\
己\end{array}$ \\
\hline 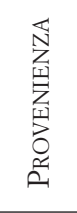 & 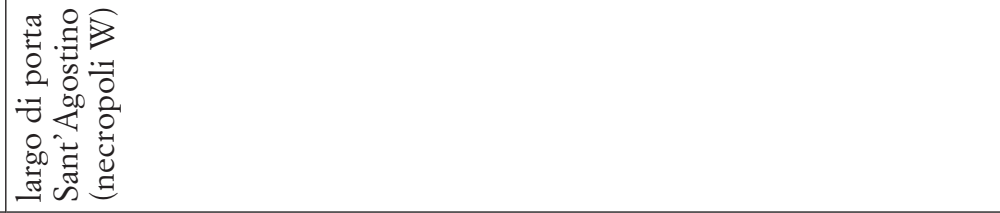 & 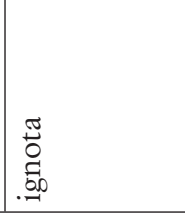 \\
\hline 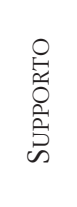 & 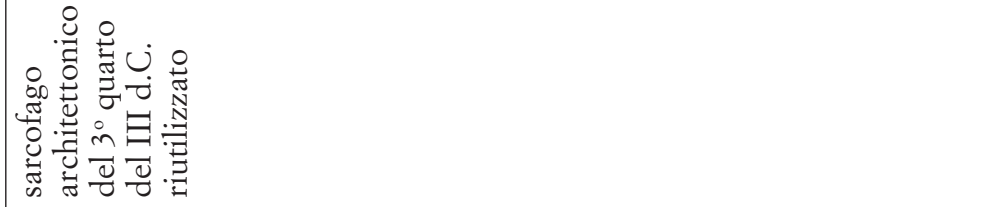 & 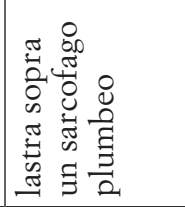 \\
\hline 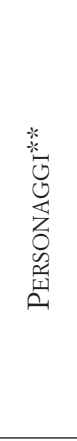 & 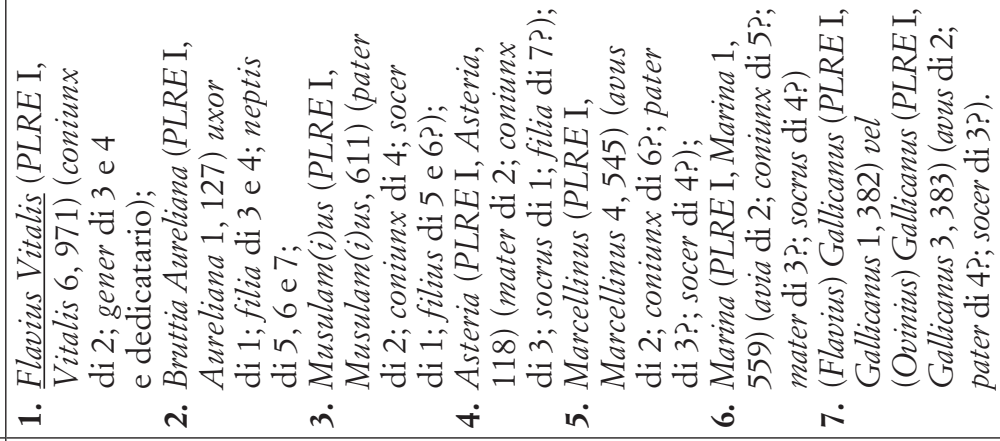 & 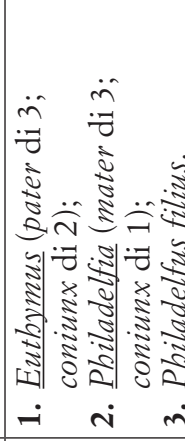 \\
\hline 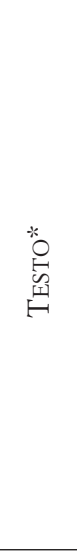 & 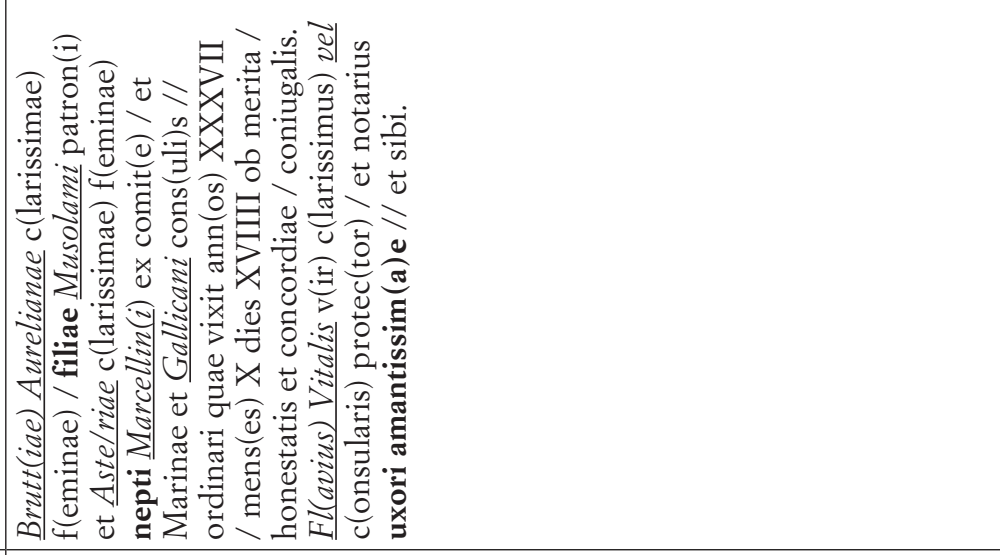 & 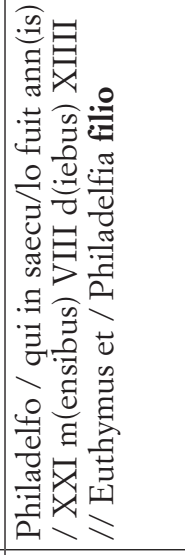 \\
\hline 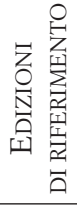 & 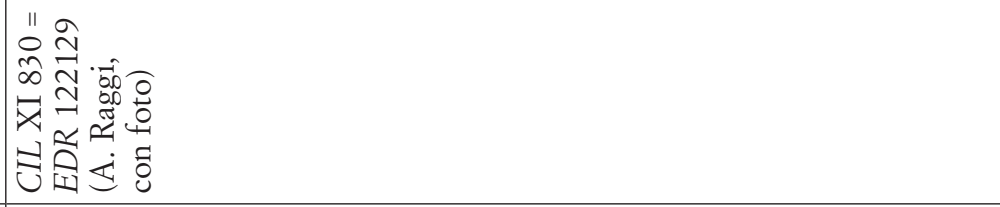 & 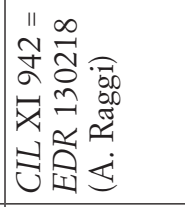 \\
\hline Z & $\Xi$ & \\
\hline
\end{tabular}


Tali documenti sono di provenienza prevalentemente urbana, benché in molti casi non sia noto l'esatto luogo di rinvenimento. L'unica eccezione è costituita da una stele in marmo proconnesio dedicata a Batonia Candida dal maritus Capellenus (Tab. 1, nr. 6) rinvenuta a Mirandola, località La Tesa in un sepolcreto da porre probabilmente in connessione con una villa rustica venuta alla luce in tale zona; quanto all'iscrizione, essa presenta anomalie grammaticali che denunciano la scarsa cultura linguistica e l'ambito popolare in cui venne concepita e si caratterizza per l'accostamento tra formule stereotipe ed espressioni più personali dei sentimenti del dedicante verso la moglie devota ${ }^{20}$.

Dall'analisi della documentazione pervenutaci si evince, tra la metà del III e la metà del IV secolo d.C., una significativa presenza di militari e di alcuni esponenti della burocrazia ufficiale, spesso di alto rango sociale, di origine modenese o legati mediante parentela a membri dell'aristocrazia locale. In particolare, nel primo gruppo si annoverano: il veteranus ex praetorio C. Maternius Quintianus, al quale dedicarono una stele funeraria la figlia Maternia Benigna e il genero M. Aurelius Maximus (Tab. 1, nr. 3); l'ex protectore [M.] Aurelius Maximianus, della cui stele è dedicante la moglie, di cui non si conserva il nome (Tab. 1, nr. 10); il vir egregius M. Aurelius Processanus, ex centurione praetoriano e protector ducenarius, per la cui sepoltura, ad opera della moglie Bononia Metrodora, venne riutilizzato un sarcofago architettonico di età severiana (Tab. 1, nr. 8). Questa pratica ovvero il reimpiego per sé o per i propri congiunti di sarcofagi in marmo proconnesio giunti non rifiniti a Mutina tra il II e III secolo d.C., forse per il tramite del porto di Ravenna - risulta ben attestata nella colonia, a partire dalla fine del III secolo d.C., per personaggi di alto rango (Tab. 1, nrr. 8, 11-14), per i quali la preziosità stessa del materiale con cui tali monumenti erano realizzati doveva costituire, in un periodo di crisi e di interruzione dei collegamenti commerciali con le cave orientali, un importante segno di distinzione sociale ${ }^{21}$. È questo il caso, ad esempio, del sarcofago architettonico del tipo Gabelmann III riutilizzato alla metà del IV secolo d.C. dal vir clarissimus, protector e notarius Flavius Vitalis per sé e per l'uxor amantissima Bruttia Aureliana (Tab. 1, nr. 14). La volontà del dedicante di attestare, nell'iscrizione sepolcrale per entrambi, gli illustri natali della moglie è evidente: nell'epigrafe ne sono infatti indicati non soltanto i genitori - ossia il notabile locale Musulam(i)us e la clarissima femina Asteria -, ma anche entrambi gli avi - un comes e un consul ordinarius - e una nonna ${ }^{22}$.

\footnotetext{
20 Calzolari 2012.

21 Rebecchi 1986, 894-895 e 918.

22 Cenerini 2014, 714-715.
} 
Il desiderio di ricordare l'insigne ascendenza della donna, allo scopo anche di dar lustro al marito e dedicante, è riscontrabile altresì su un altro monumento funerario modenese: si tratta di un sarcofago della metà del III secolo d.C. riutilizzato negli anni '20 del secolo successivo dal vir consularis L. Nonius Verus, probabilmente originario di Mutina ${ }^{23}$, per la moglie - la clarissima femina Vinicia Marciana -, figlia del perfectissimus vir Caecilianus, del quale viene ricordata, limitatamente alle ultime cinque e più prestigiose cariche, la carriera ${ }^{24}(T a b .1, n r .12 a)$. Chiara è la funzione onoraria per il marito-committente, il cui nome compare nella parte iniziale dell'iscrizione seguito dal cursus; la definizione della defunta come coniunx sanctissima ac benignissima e l'esaltazione delle sue virtù ben si inseriscono anch'esse in una logica di autorappresentazione aristocratica, in cui le tradizionali doti matronali accrescevano la dignitas degli uomini della famiglia ${ }^{25}$. Vinicia pare inoltre aver assolto il principale compito femminile, ovvero dare una discendenza al marito: su uno dei fianchi del sarcofago, andato perduto, compaiono infatti le dediche, realizzate in due momenti successivi, al clarissimus puer L. Nonius Faustinus e alla clarissima femina Nonia Laudicia, evidentemente premorti al padre (Tab. 1, nr. 12b).

L. Nonius Verus compare come committente di un'altra iscrizione modenese incisa su un sarcofago a cassapanca riutilizzato della seconda metà del II secolo d.C., rinvenuto all'incrocio tra Corso Cavour e via Ganaceto: si tratta dell'epigrafe sepolcrale o commemorativa della morte di L. Peducea Iuliana, probabile figlia di un notabile locale ${ }^{26}$, scomparsa a poco più di tredici anni dopo soli cinque mesi e venti giorni di matrimonio (Tab. 1, nr. 11). L'iscrizione è assai poco accurata nella realizzazione: il ductus è molto irregolare, con lettere talora di dimensioni assai inferiori per un probabile calcolo errato degli spazi epigrafici; alcune lettere sono erroneamente omesse; nell'onomastica della dedicataria è presente il praenomen; l'indicazione memori(a)e cla(rissimae) fem(inae) è incisa al di fuori dello specchio epigrafico, in uno spazio ricavato dall'asportazione di parte del listello inferiore della cornice superiore della fronte dell'arca. Questi elementi, associati alla constatazione che l'iscrizione è incompleta nell'ultima riga - che avrebbe probabilmente dovuto prevedere il completamento del cognomen del dedicante L. Nonius Ver(us), forse il suo status sociale ed il rapporto che lo legava alla dedicataria - fanno sorgere qualche dubbio

23 Tale ipotesi, proposta da S. Panciera (Panciera 1987, 86), è in contrasto con quella di F. Rebecchi, secondo il quale L. Nonius Verus sarebbe stato il discendente di una gens veronese ma originaria di Brescia (Rebecchi 1986, 917).

24 Porena 2006, 138.

25 Cenerini 2014, 714.

26 Cenerini 2014, 710-711. 
in merito all'effettivo utilizzo del sarcofago, che pare però confermato dal fatto che esso venne rinvenuto in un'area di necropoli nel suburbio N-W della città, presso la porta urbica di Ganaceto ${ }^{27}$. Nel caso in cui sia stato effettivamente messo in opera, questo disinteresse per il completamento dell'epigrafe potrebbe essere conseguente ad un nuovo matrimonio contratto da L. Nonius Verus - forse proprio con Vinicia Marciana - in breve tempo, anche alla luce del fatto che l'unione con Peducea, vista la brevità, fu certamente priva di prole. In alternativa, è stato recentemente proposto da F. Cenerini che la menzione del clarissimato della defunta, incisa al di fuori della tabula ansata, sia stata apposta in un momento successivo alla deposizione della donna, forse in concomitanza con la sepoltura di Vinicia, clarissima femina in quanto moglie di un vir consularis, al fine di dare ulteriore lustro allo stesso senatore. Anche lo spazio epigrafico ottenuto dal ritaglio della cornice inferiore al di sotto dell'ultima linea, occupata solo parzialmente dal nome del dedicante, potrebbe essere stato ricavato nello stesso momento allo scopo di ospitare la trascrizione, forse in forma abbreviata, del cursus di L. Nonius Verus, rimasta però incompiuta ${ }^{28}$.

Il nome di L. Nonius Verus è attestato, inoltre, sulla fronte di un sarcofago della seconda metà del III secolo d.C., reimpiegato entro la metà del secolo successivo, di cui si conservano alcuni frammenti nel Museo Campanini di Canossa, in quanto fu riutilizzato da un antenato della contessa Matilde; in esso il personaggio compare, insieme alla moglie Sulpicia Triaria, come dedicante dell'iscrizione funeraria ai suoceri C. Sulpicius Agatangelus e Vibia Vibiana (Tab. 1, nr. 13) ed è indicato semplicemente come gener, senza alcun riferimento al suo status sociale né al cursus.

Un'identificazione del L. Nonius Verus menzionato su quest'ultimo monumento col vir consularis attestato sui due sarcofagi modenesi è stata ampiamente accettata, suscitando il problema della cronologia dei suoi tre matrimoni. In particolare, F. Rebecchi ha ipotizzato il seguente «ordine» delle mogli, ripreso anche nelle schede EDR relative alle tre iscrizioni, senza però dare giustificazione alcuna: Peducea Iuliana, Vinicia Marciana e Sulpicia Triaria ${ }^{29}$. Recentemente F. Cenerini ha invece proposto una successione diversa: il primo matrimonio sarebbe stato quello con Sulpicia Triaria, l'unica a non possedere il titolo di clarissima femina, dal momento che L. Nonius Verus è indicato in qualità di codedicante del sarcofago dei suoceri con la sola onomastica; il secondo quello con Peducea Iuliana, morta prematuramente senza prole; il terzo il più illustre, ovvero quello

27 Parra 1988.

28 Cenerini 2014, 713.

29 Rebecchi 1986, 918-921. 
con Vinicia Marciana, figlia di un funzionario degli imperatori Massimiano e Massenzio, e l'unica ad aver garantito sicuramente una discendenza al marito, benché i figli - o almeno due di essi - fossero premorti al padre ${ }^{30}$.

In realtà, se certi sono la provenienza modenese per i sarcofagi di $\mathrm{Pe}$ ducea e Vinicia così come il fatto che il matrimonio con la figlia dell'illustre Caecilianus fu posteriore a quello con la giovane Iuliana, qualche dubbio permane sull'effettiva identificazione col vir consularis del L. Nonius Verus ricordato sul terzo sarcofago. Sospetta potrebbe essere anzitutto la totale assenza di riferimenti allo status sociale e alla carriera del personaggio; inoltre, benché le caratteristiche tipologiche del sarcofago e il legame col consolare L. Nonius Verus ne abbiano fatto ipotizzare, sin dal Bormann, una provenienza modenese ${ }^{31}$, l'assenza di notizie in merito al rinvenimento o a un suo riutilizzo successivo a Modena non ne esclude un'origine diversa. A tal proposito C. Corti ha ipotizzato, pur accettando un'identificazione del personaggio col vir consularis, che tale monumento fosse collocato in un'area necropolare legata alla frequentazione del vicus di Luceria, individuato presso Ciano d'Enza (RE), sede di un forum pecuarium, a conferma del ben noto coinvolgimento della gens Nonia di Mutina nella lavorazione e nella vendita delle stoffe di lana. Un legame con tale attività parrebbe ravvisarsi, d'altronde, anche nel cursus di L. Nonius Verus, dal momento che in tutte le località in cui esercitò i suoi incarichi, o il patronato, l'allevamento e la produzione tessile rappresentavano uno dei settori trainanti dell'economia ${ }^{32}$. Alla luce di tali considerazioni non pare comunque da escludere che il marito di Sulpicia Triaria fosse un omonimo o, più probabilmente, un congiunto del consolare L. Nonius Verus.

\section{RIFLESSIONI CONCLUSIVE}

L'analisi delle testimonianze epigrafiche modenesi consente, per il periodo in oggetto, di ricostruire con facilità, vista l'esplicita menzione dei legami di parentela tra i personaggi citati nelle iscrizioni, vari nuclei familiari, talora, come nel caso del sarcofago di Bruttia Aureliana, comprensivi anche degli ascendenti sino alla seconda generazione; per il vir consularis L. Nonius Verus, come visto, è possibile addirittura ricostruire più nuclei familiari, connessi ai vari matrimoni - due o tre - contratti da costui.

30 Cenerini 2014, 712-713.

31 Si veda da ultimo Franzoni 2008.

32 Corti 2012, 218-219. 
Fanno eccezione unicamente due documenti: il primo di essi è l'iscrizione sepolcrale alla domina Aurelia Agathonice da parte del servus Sabinianus, che, pur non riferendosi ad un vincolo parentale, rientra comunque nell'ambito dei rapporti interni alla familia romana (Tab. 1, nr. 2). Il secondo è il monumento funerario, noto solo dal CIL, offerto da Aurelia Maxima al marito M. Aurelius Maximus, ai figli e ai nipoti nonché al veteranus Aurelius Tzinius (Tab. 1, nr. 2); il fatto che, nell'edizione del Bormann, il soggetto della dedica sia al singolare, Aurelia Maxima, mentre il verbo è al plurale, curaverunt, parrebbe sottintendere una partecipazione nella dedica del veterano - probabilmente ancora vivente vista la mancanza dei dati biometrici presenti invece per gli altri dedicatari - in qualità forse di congiunto della dedicante o del marito defunto.

Degna di menzione è, infine, l'iscrizione su sarcofago fatta realizzare dal filius Sosius Ptolemaeus alla mater Sosia Q. f. Herennia e al di lei alumnus Sosius Felicianus (Tab. 1, nr. 7). La constatazione che il dedicante porta lo stesso gentilizio della madre, che è un'ingenua in quanto nella sua onomastica compare il patronimico, potrebbe far ipotizzare che il padre fosse anch'egli un membro della gens Sosia o un liberto della stessa ${ }^{33}$. Più interessante risulta la posizione dell'altro dedicatario, definito alumnus di Sosia, termine col quale, secondo l'interpretazione di H.S. Nielsen, si indicherebbe un rapporto basato principalmente sull'affetto che assumeva i caratteri di una quasi-adozione, non sancita a quanto pare da alcun vincolo legale; tale legame era duraturo ed indipendente da altre relazioni sociali ad esempio il matrimonio o la paternità/maternità - intessute da una delle due parti e implicava, per l'affidatario, un senso di responsabilità in merito al futuro economico del protetto ${ }^{34}$.

\section{BIBLIOGRAFIA}

Calzolari 1984

M. Calzolari, Carta degli insediamenti di età romana nella Bassa Modenese (Comuni di Mirandola, San Felice sul Panaro e Finale Emilia), Modena 1984.

Calzolari 2012

M. Calzolari, La stele funeraria di «Batonia Candida», in M. Calzolari - F. Foroni (a cura di), L'insediamento romano della Tesa di Mirandola (MO). Ricognizioni e scavi 1930-2011 (Quaderni di Archeologia dell'Emilia Romagna 33), Firenze 2012, 215-217.

33 Quest'ultima ipotesi parrebbe la più probabile visto il cognomen grecanico del dedicante.

34 Nielsen 1987, 142-143 e 187-188. 
Campagnoli 2006

Cattani 1998

Cenerini 2014

Corti 2012

Franzoni 2008

Gelichi - Malnati - Ortalli 1986

Giordani 2000

Giordani 2005

Giordani - Ricci 2005

Jacques 1983

Nielsen 1987

Panciera 1987
P. Campagnoli, Le vie da Modena, in P.L. Dall'Aglio I. Di Cocco (a cura di), La linea e la rete. Formazione storica del sistema stradale in Emilia-Romagna, Milano 2006, 180-191.

M. Cattani, Stratificazione e centri storici: il caso di Modena, in S. Gelichi (a cura di), Archeologia medievale in Emilia occidentale. Ricerche e studi (Documenti di Archeologia 15), Mantova 1998, 35-49.

F. Cenerini, La rappresentazione epigrafica delle «clarissimae feminae» a «Mutina»: qualche spunto di riflessione, in M.L. Caldelli - G.L. Gregori (a cura di), Epigrafia e ordine senatorio, 30 anni dopo (Tituli 10), Roma 2014, 709-719.

C. Corti, L'economia della lana a «Mutina», in M.S. Busana - P. Basso (a cura di), La lana nella Cisalpina. Economia e società (Antenor Quaderni 27), Padova 2012, 213-229.

C. Franzoni, Frammenti del sarcofago di «C. Sulpicius Agatangelus», reimpiegato come sepoltura degli avi di Matilde di Canossa, in A. Calzona (a cura di), Matilde e il tesoro dei Canossa, tra castelli, monasteri e città, Cinisello Balsamo (MI) 2008, 447-448.

S. Gelichi - L. Malnati - J. Ortalli, L'Emilia centrooccidentale tra la media età imperiale e l'alto Medioevo, in A. Giardina (a cura di), Società romana e impero tardo antico, III, Le merci, gli insediamenti, Bari 1986, 543-645.

N. Giordani, Modena, in M. Marini Calvani (a cura di), «Aemilia». La cultura romana in Emilia Romagna dal III secolo a.C. all'età costantiniana, Venezia 2000, 423-434.

N. Giordani, Sarcofago a cassapanca, in N. Giordani - G. Paolozzi Stozzi (a cura di), Il Museo Lapidario Estense. Catalogo generale, Venezia 2005, 135.

N. Giordani - M. Ricci, Stele funeraria, in N. Giordani - G. Paolozzi Stozzi (a cura di), Il Museo Lapidario Estense. Catalogo generale, Venezia 2005, 149-150.

F. Jacques, Les curateurs des cités dans l'Occident romain de Trajan à Gallien. Études prosopographiques, Paris 1983.

H.S. Nielsen, «Alumnus»: A Term of Relation Denoting Quasi-adoption, CEM 38 (1987), 141-188.

S. Panciera, I patroni di Aquileia tra la città e Roma, in Aquileia e Roma, e indici dal vol. XXI al XXX (Antichità Alto Adriatiche 30), Udine 1987, 77-95. 
Parra 1988

Porena 2006

Rebecchi 1969

Rebecchi 1986

Sastre de Diego 2011
C. Parra, Carta Archeologica urbana, Scheda n. 68, in Modena dalle origini all'anno Mille. Studi di archeologia e storia, II, Modena 1988, 370-371.

P. Porena, L'Italia prima di Ponte Milvio e la carriera di «Caecilianus», Epigraphica 68 (2006), 117-154.

F. Rebecchi, Aggiornamento epigrafico modenese, AttiMod 4 (1969), 261-278.

F. Rebecchi, Appunti per una storia di Modena nel tardo-impero: monumenti e contesto sociale, MEFRA 98 (1986), 881-930.

I. Sastre de Diego, La reutilización de epígrafes romanos en la Hispania tardoantigua: los altares cristianos y la polémica ara de Guadix, in J. Carbonell Manils H. Gimeno Pascual - J.L. Moralejo Álvarez (eds.), El monumento epigráfico en contextos secundarios. Procesos de reutilización, interpretación y falsificación, Barcelona 2011, 67-87.

\section{Abbreviazioni}

$A E ́$

L'Année épigraphique: Revue des publications épigraphiques relatives à l'antiquité romaine, Paris 1888-.

CIL

$E D R$

$I L C V$

$P^{2} R^{2}$

PLRE
Corpus Inscriptionum Latinarum, consilio et auctoritate Academiae litterarum regiae Borussicae editum, Berlin 1863-.

Epigraphic Database Rome, http://www.edr-edr.it/ Italiano/index_it.php.

E. Diehl, Inscriptiones Latinae Christianae Veteres, Berolini, poi Dublin - Zürich 1925-1967.

Prosopographia Imperii Romani saec. I, II, III, editio altera, edd. E. Groag - E. Stein - L. Petersen, I-, Berlin 1933-.

The Prosopography of the Later Roman Empire, edd. A.H.M. Jones - J.R. Martindale - J. Morris, Cambridge 1971-1992. 